\title{
Clinical and Genetic Risk Factors Associated With the Presence of Lupus Nephritis
}

Jung-Min Shin, M.D. ${ }^{1}$, Dam Kim, M.D., Ph.D. ${ }^{2}$, Young-Chang Kwon, Ph.D. ${ }^{3}$, Ga-Young Ahn, M.D. ${ }^{4}$, Jiyoung Lee, M.S. ${ }^{3}$, Youngho Park, Ph.D. ${ }^{5}$, Yeon-Kyung Lee, M.D. ${ }^{1}$, Tae-Han Lee, M.D. ${ }^{1}$, Dae Jin Park, M.D. ${ }^{1}$, Yeo-Jin Song, M.D. ${ }^{1}$, Eunji Ha, B.S. ${ }^{6,7}$, Kwangwoo Kim, Ph.D. ${ }^{6,7}$, So-Young Bang, M.D., Ph.D. ${ }^{1,3}$ Chan-Bum Choi, M.D., Ph.D. ${ }^{1,3}$, Hye-Soon Lee, M.D., Ph.D. ${ }^{1,3}$, Sang-Cheol Bae, M.D., Ph.D., MPH ${ }^{1,3}$

${ }^{1}$ Department of Rheumatology, Hanyang University Hospital for Rheumatic Diseases, Seoul, ${ }^{2}$ Department of Rheumatology, Myongji Hospital, Hanyang University College of Medicine, Goyang, ${ }^{3}$ Hanyang University Institute for Rheumatology Research, Seoul, ${ }^{4}$ Division of Rheumatology, Department of Internal Medicine, Korea University Guro Hospital, Seoul, ${ }^{5}$ Department of Big Data Application, College of Social Economic \& Interdisciplinary Studies, Hannam University, Daejeon, Departments of ${ }^{6}$ Life and Nanopharmaceutical Sciences, ${ }^{7}$ Biology, Kyung Hee University, Seoul, Korea

Objective. To elucidate whether clinical features and the weighted genetic risk score (wGRS) were associated with the presence of lupus nephritis (LN). Methods. We retrospectively divided patients with systemic lupus erythematosus $(\mathrm{SLE}, \mathrm{n}=1,078)$ into biopsy-proven $L N(n=507)$ and non-LN groups (non-LN, $n=571)$. Baseline clinical features, serologic markers, and the wGRS were collected. The wGRS was calculated from 112 non-human leukocyte antigen (non-HLA) loci and HLA-DR $\beta 1$ amino acid haplotypes for SLE. Associations among clinical features, wGRS, and the presence of LN were identified. Results. In the multivariate analysis, patients with $L N$ were younger at diagnosis (odds ratio $[O R]=0.97, p<0.001)$, had more pleuritis $(O R=2.44$, $\mathrm{p}<0.001)$ and pericarditis $(O R=1.62, p=0.029)$, had a higher detection rate of anti-double stranded deoxyribonucleic acid (anti-dsDNA antibodies, OR =2.22, $\mathrm{p}<0.001$ ), anti-Smith antibodies (anti-Sm antibodies, $\mathrm{OR}=1.70, \mathrm{p}=0.002$ ), low level of complement $(O R=1.37, p=0.043)$ and absence of antiphospholipid antibodies (aPL antibodies, $O R=1.60, p=0.002)$, and had higher wGRS (OR =1.16, $p=0.012)$. Mediation analysis suggested that anti-Sm antibodies and low complement could be mediators in the relationship between high wGRS and the presence of LN. Conclusion. Onset age, pleuritis, pericarditis, several serologic markers, and wGRS were associated with the presence of LN. Anti-Sm antibodies and low complement appeared to mediate the indirect relationship between wGRS and the presence of LN. (J Rheum Dis 2021;28:150-158)

Key Words. Systemic lupus erythematosus, Lupus nephritis, Genetic risk score, Associated factors

\section{INTRODUCTION}

Systemic lupus erythematosus (SLE) is a heterogeneous autoimmune disease well known for its multisystemic presentation, ranging from cutaneous manifestations to vital organ disorders [1]. Lupus nephritis (LN) is one frequent and severe organ manifestation of SLE [2] that affects $12 \% \sim 69 \%$ of patients with SLE [3]. Despite current advanced treatments, LN constitutes a major cause of renal failure and is associated with reduced long-term sur- vival [3]. Early diagnosis and treatment with immunosuppressive agents are important for improving outcomes associated with LN [4]. Thus, recognizing patients at risk for $\mathrm{LN}$ is advantageous for early diagnosis and prompt intervention with immunosuppressive agents, thereby reducing the mortality risk.

Previous studies have suggested that several clinical and immunologic features, such as age, sex, hypertension, malar rash, anemia, anti-double stranded deoxyribonucleic acid antibodies (anti-dsDNA antibodies), anti-Smith an-

Received : December 22, 2020, Revised : April 30, 2021, Accepted : May 3, 2021

Corresponding to : Sang-Cheol Bae (iD http://orcid.org/0000-0003-4658-1093

Department of Rheumatology, Hanyang University Hospital for Rheumatic Diseases, 222 Wangsimni-ro, Seongdong-gu, Seoul 04763, Korea. E-mail : scbae59@gmail.com

Copyright (c) 2021 by The Korean College of Rheumatology.

This is an Open Access article, which permits unrestricted non-commerical use, distribution, and reproduction in any medium, provided the original work is properly cited. 
tibodies (anti-Sm antibodies), low complement, lupus anticoagulant [5-7], and genetic factors [8] are associated with LN. Familial and twins studies have elucidated the genetic associations of SLE [9], and large-scale metaanalyses of genome-wide association studies (GWASs) have determined a significant association between common genetic variants and disease risk for SLE $[10,11]$. A genetic risk score (GRS) effectively calculates the estimated effect of genetic risk on disease susceptibility, aggregating the risk loci determined in GWASs into a single measurement [12]. The weighted genetic risk score (wGRS) reflects how frequently the risk alleles were found in patients' genomes using an allelic odds ratio (OR) in a logarithmic scale [13]. A recent study demonstrated that the individual GRS for SLE can predict early disease onset and damage accrual including LN [8]. We investigated the ability of genetic risk to predict presence of LN using the wGRS calculated from the recently reported 112 non-human leukocyte antigen (non-HLA) loci and HLA-DR $\beta 1$ amino acid haplotypes [11].

Here, we investigated clinical and genetic features significantly associated with $\mathrm{LN}$. Genetic risk was estimated using wGRS.

\section{MATERIALS AND METHODS}

\section{Patients}

All patients who fulfilled the American College of Rheumatology (ACR) classification criteria for SLE [14] were enrolled. All subjects provided written informed consent for inclusion in the Hanyang BAE Lupus cohort (BAE Registry of Autoimmune Diseases for Epidemiology) [15], a Korean single-center prospective observational cohort for which clinical information was updated annually from 1998 2018. This study protocol was approved by the Institutional Ethics Review Board of Hanyang University Hospital (IRB no: HYUH2001-06-001).

All patients with SLE were retrospectively classified into two groups based on renal involvement. All patients with LN were confirmed by renal biopsy before enrollment or during the follow-up period. Those who never met the ACR renal disorder criteria $[14,16]$ before enrollment and during the follow-up period were classified as patients without LN (non-LN). Patients who met the ACR classification criteria for renal disorder but who lacked renal biopsy results were excluded from this study. A flow diagram of this study is summarized in Figure 1.

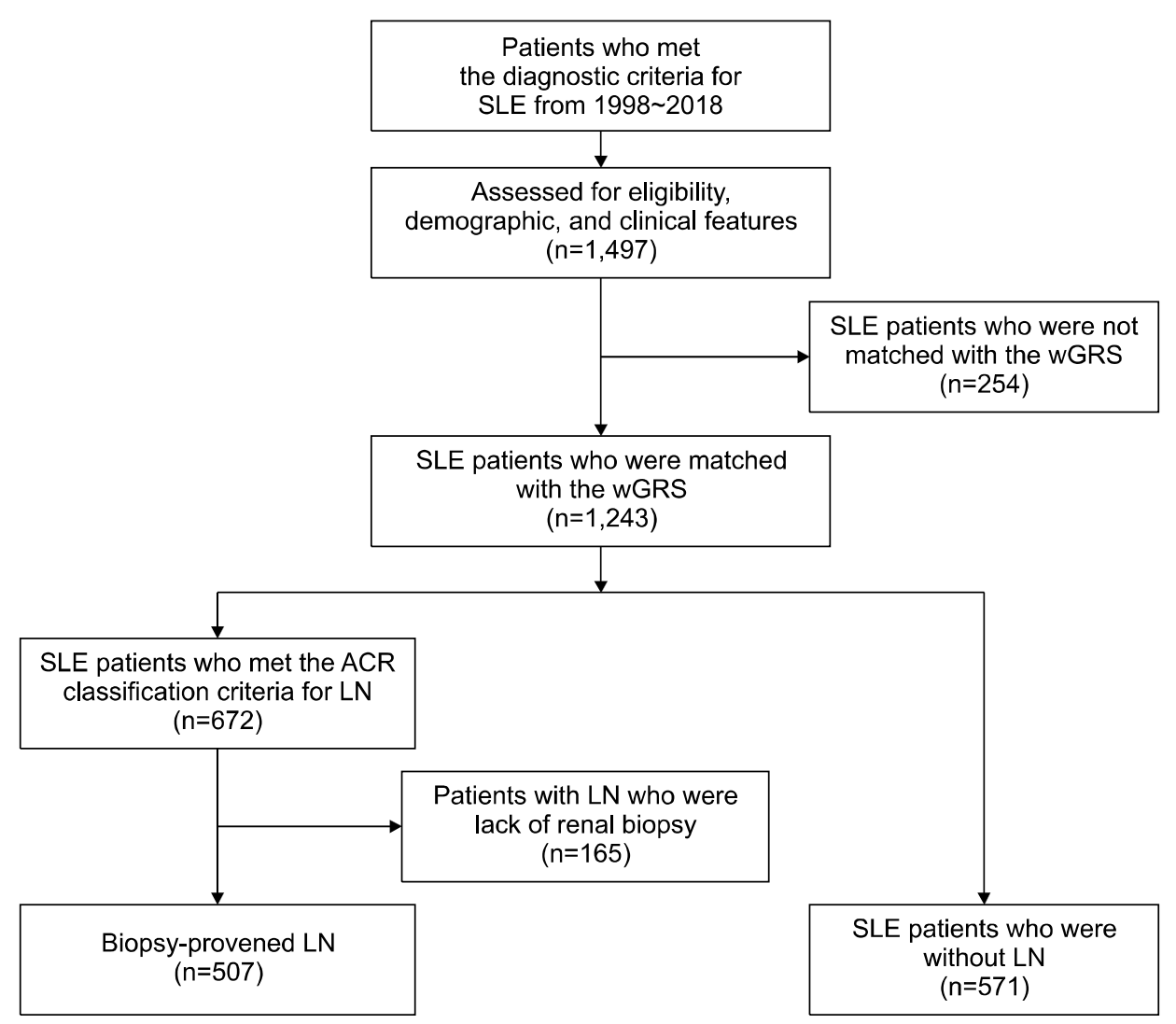

Figure 1. Flow diagram of the study design. SLE: systemic lupus erythematosus, wGRS: weighted genetic risk score, ACR: American College of Rheumatology, LN: lupus nephritis. 


\section{Clinical characteristics}

Demographic and socioeconomic data were collected at enrollment. Clinical features such as the SLE Disease Activity Index-2000 [17], Systemic Lupus International Collaborating Clinics/ACR Damage Index (SDI) [18], complete blood count, chemistry, immunology, and urinalysis results were recorded and followed up at least annually.

Until 2004, histological classification of biopsy findings was based on the 1982 World Health Organization (WHO) classification for LN [19]; then, the International Society of Nephrology/Renal Pathology Society (ISN/RPS) classification was adopted [20]. Activity and chronicity indices $[21,22]$ were also collected. If a patient with LN had undergone more than two renal biopsies, we selected the first renal biopsy result for analysis.

\section{Calculation of the wGRS}

The GRS was evaluated to elucidate its genetic effect on LN in patients with SLE. The GRS was weighted according to effect size based on the allelic odds ratio of each variant from previously reported SLE-risk loci [13], which constitute the 112 non-HLA loci of the most updated East Asian study [11] and the HLA-DR $\beta 1$ haplotypes in amino acid positions 11, 13, and 26 [23]. Finally, we used the wGRS of each patient to predict the presence of $\mathrm{LN}$.

\section{Statistical analyses}

We used Student's t-test for continuous variables and the chi-square test for categorical variables of demographics, clinical characteristics, laboratory findings, and wGRS to compare the differences between the LN and non-LN groups. Logistic regression analysis was conducted to determine the associations among the demographics, clinical features, wGRS, and the presence of LN. All analyses were performed using the SAS 9.2 statistical software (SAS Institute, Cary, NC, USA). All tests were two-sided, and p-values $<0.05$ were considered statistically significant.

Mediation analysis has been commonly used in sociology, epidemiology [24], and clinical fields to evaluate coronary artery disease and osteoarthritis [25,26]. This method has been adopted for its ability to identify the causal relationship between an exposure and an outcome through mediators. In this study, among all of the associated clinical features of LN determined in the logistic regression analysis, we focused on serologic markers such as anti-Sm antibodies and low complement, which are ob- jective and less affected by inter- and intrarater reliability, and assumed that they were mediators. We defined a three-step relationship among the exposure, mediators, and outcome (Figure 2). Step one was to determine the association between the wGRS and serologic markers, step two was to determine the association between the wGRS and LN, and step three was to determine the association between serologic markers and LN. If a variable was confirmed to be a mediator, all three steps should be statistically significant [27]. Furthermore, the significance of step three should be decreased with the addition of a mediator [24]. We sequentially performed logistic regression analyses on all three steps and conducted Sobel's test [27] to recertify the hypothesis. In Sobel's test statistics, absolute values over \pm 1.96 were considered statistically significant.

\section{RESULTS}

\section{Differences in demographics and clinical manifestations}

A total of 1,078 patients were enrolled in this study, and all patients were of a single ethnic origin. The study population was predominantly female $(n=995,92.3 \%)$. LN was diagnosed in 507 (47.0\%) patients, and 571 subjects were included in the non-LN group $(53.0 \%)$ during a

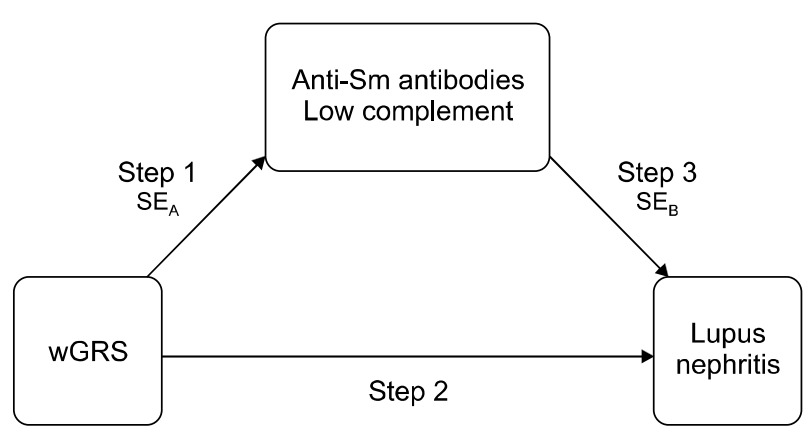

Figure 2. Conceptual model on three-step relationship between the exposure, mediator, and outcome. Step 1: association between the wGRS and serologic markers calculated by logistic regression analysis, step 2: association between the wGRS and lupus nephritis (LN) calculated by logistic regression analysis, step 3: association between serologic markers and LN calculated by logistic regression analysis, Sm: Smith, SEA: associated standard error for step 1 calculated by Sobel's test, SEB: associated SE for step 3 calculated by Sobel's test, SEA for anti-Sm antibodies: 0.066, SEA for low complement: 0.057, SEB for anti-Sm antibodies: 0.157, SEB for low complement: 0.141 . 
mean follow-up period of $10.4 \pm 6.1$ years. Table 1 shows the clinical features based on the ACR SLE classification criteria [14] and the wGRS in the biopsy- proven LN and non-LN groups. There were fewer female patients with SLE in the LN group than in the non-LN group $(n=457$, $90.1 \%$ in the $\mathrm{LN}$ group vs. $\mathrm{n}=538,94.2 \%$ in the non- $\mathrm{LN}$ group, $\mathrm{p}=0.017)$. Patients in the SLE group were typically diagnosed with SLE at a younger age $(25.4 \pm 10.2$ years vs. $29.4 \pm 11.0$ years, $p<0.001$ ) and had a longer duration between SLE diagnosis and enrollment than those in the non-LN group $(3.2 \pm 4.1$ years vs. $2.4 \pm 3.4$ years, $\mathrm{p}<$ $0.001)$. Among the clinical features, the presence of a ma-

Table 1. Comparison of baseline clinical features and wGRS in patients with lupus nephritis and non-lupus nephritis

\begin{tabular}{|c|c|c|c|c|}
\hline Variable & $\begin{array}{l}\text { All patients } \\
(\mathrm{n}=1,078)\end{array}$ & $\begin{array}{c}\mathrm{LN} \\
(\mathrm{n}=507)\end{array}$ & $\begin{array}{l}\text { Non-LN } \\
(n=571)\end{array}$ & p-value \\
\hline Sex, female & $995(92.3)$ & $457(90.1)$ & $538(94.2)$ & 0.017 \\
\hline Age at diagnosis of SLE (yr) & $28.0 \pm 10.8$ & $25.4 \pm 10.2$ & $29.4 \pm 11$ & $<0.001^{*}$ \\
\hline Duration between SLE diagnosis and enrollment (yr) & $2.8 \pm 3.7$ & $3.2 \pm 4.1$ & $2.4 \pm 3.4$ & $<0.001^{*}$ \\
\hline wGRS & $22.8 \pm 1.2$ & $22.9 \pm 1.1$ & $22.6 \pm 1.2$ & $<0.001^{*}$ \\
\hline Malar rash & $467(43.4)$ & $247(48.7)$ & $220(38.6)$ & 0.001 \\
\hline Discoid rash & $84(7.8)$ & $33(6.5)$ & $51(8.9)$ & 0.172 \\
\hline Photosensitivity & $370(34.5)$ & $164(32.4)$ & $206(36.3)$ & 0.288 \\
\hline Oral ulcer & $372(34.5)$ & $166(32.8)$ & $206(36.1)$ & 0.196 \\
\hline Arthritis & $698(64.8)$ & $324(63.9)$ & $374(65.6)$ & 0.602 \\
\hline Serositis & $244(22.7)$ & $160(31.6)$ & $84(14.7)$ & $<0.001$ \\
\hline Pleuritis & $185(17.2)$ & $127(25.1)$ & $58(10.2)$ & $<0.001$ \\
\hline Pericarditis & $145(13.5)$ & $96(19.0)$ & $49(9.0)$ & $<0.001$ \\
\hline Neurologic disorder & $48(4.5)$ & $24(4.7)$ & $24(4.2)$ & 0.768 \\
\hline Immunologic disorder & $934(86.8)$ & $466(92.0)$ & $468(82.3)$ & $<0.001$ \\
\hline Anti-dsDNA antibodies & $838(77.8)$ & $436(86.0)$ & $402(70.6)$ & $<0.001$ \\
\hline Anti-Sm antibodies & 207 (19.2) & $124(24.5)$ & $83(14.6)$ & $<0.001$ \\
\hline Low complement & 791 (73.4) & $399(78.7)$ & $392(68.7)$ & $<0.001$ \\
\hline aPL antibodies & $321(30.0)$ & $133(26.2)$ & $188(33.0)$ & 0.018 \\
\hline Hematologic disorder & $885(82.3)$ & $428(84.6)$ & $457(80.2)$ & 0.070 \\
\hline Hemolytic anemia & $163(15.1)$ & $89(17.6)$ & $74(13.0)$ & 0.045 \\
\hline Leukopenia & $638(59.3)$ & $301(59.5)$ & $337(59.1)$ & 0.953 \\
\hline Lymphopenia & $686(63.8)$ & $341(67.4)$ & $345(60.5)$ & 0.023 \\
\hline Thrombocytopenia & $259(24.0)$ & $139(27.4)$ & $120(21.0)$ & 0.017 \\
\hline ANA & $1,078(100)$ & $507(100)$ & $571(100)$ & - \\
\hline Total number of ACR criteria & $5.2 \pm 1.4$ & $5.8 \pm 1.4$ & $4.7 \pm 1.2$ & $<0.001^{*}$ \\
\hline SDI & $0.3 \pm 0.7$ & $0.4 \pm 0.9$ & $0.3 \pm 0.6$ & $0.001 *$ \\
\hline SDI, non-renal & $0.3 \pm 0.6$ & $0.3 \pm 0.7$ & $0.3 \pm 0.6$ & $0.437^{*}$ \\
\hline \multicolumn{5}{|l|}{ Renal biopsy classification } \\
\hline $\mathrm{II}+\mathrm{V}$ & & $2(0.4)$ & & \\
\hline III & & $106(20.9)$ & & \\
\hline $\mathrm{III}+\mathrm{V}$ & & $68(13.4)$ & & \\
\hline IV & & $226(44.6)$ & & \\
\hline $\mathrm{IV}+\mathrm{V}$ & & $30(5.9)$ & & \\
\hline $\mathrm{V}$ & & $75(14.8)$ & & \\
\hline Activity index, $\mathrm{n}=312^{\dagger}$ & & $7.0 \pm 4.2$ & & \\
\hline Chronicity index, $\mathrm{n}=312^{\dagger}$ & & $1.7 \pm 1.6$ & & \\
\hline
\end{tabular}

Values are presented as mean \pm standard deviation, or number (\%). LN: systemic lupus erythematosus patients with lupus nephritis, non-LN: systemic lupus erythematosus patients without LN, SLE: systemic lupus erythematosus, wGRS: weighted genetic risk score, dsDNA: double-stranded deoxyribonucleic acid, Sm: Smith, aPL: antiphospholipid, ANA: antinuclear antibodies, ACR: American College of Rheumatology, SDI: Systemic Lupus International Collaborating Clinics/ACR Damage Index total score at enrollment, SDI, non-renal: total score of SDI at enrollment except for the variables related to LN; such as proteinuria, pyuria, hematuria, and abnormal urinary casts. ${ }^{*}$ p-values are calculated by Student's t-test and the chi-square test. $p$-values $<0.05$. ${ }^{\dagger}$ Numbers are reduced due to lack of data. 
lar rash ( $\mathrm{n}=247,48.7 \%$ vs. $\mathrm{n}=220,38.6 \%, \mathrm{p}=0.001)$ and serositis ( $n=160,31.6 \%$ vs. $n=84,14.8 \%, p<0.001)$ and immunologic abnormalities including anti-dsDNA $(\mathrm{n}=436,86.0 \%$ vs. $\mathrm{n}=402,70.6 \%, \mathrm{p}<0.001)$, anti-Sm antibodies ( $n=124,24.5 \%$ vs. $n=83,14.6 \%, p<0.001)$, low complement $(n=399,78.7 \%$ vs. $n=392,68.7 \%$, $\mathrm{p}<0.001$ ), and negative antiphospholipid antibodies (aPL antibodies, $\mathrm{n}=133,26.2 \%$ vs. $\mathrm{n}=188,33 \%$, $\mathrm{p}=0.018$ ) occurred more frequently in the $\mathrm{LN}$ group than in the non-LN group. In addition, with regard to hematologic disorders, hemolytic anemia ( $\mathrm{n}=89,17.6 \%$ vs. $\mathrm{n}=74,13 \%, \mathrm{p}=0.045)$, lymphopenia $(\mathrm{n}=341,67.4 \%$ vs. $\mathrm{n}=345,60.5 \%, \mathrm{p}=0.023)$, and thrombocytopenia ( $\mathrm{n}=$ $139,27.4 \%$ vs. $n=120,21.0 \%, p=0.017$ ) were more frequently reported in the $\mathrm{LN}$ group than in the non-LN group. The wGRS was higher in patients in the LN group than in those in the non-LN group ( $22.9 \pm 1.1$ vs. $22.6 \pm$ $1.2, \mathrm{p}<0.001)$. The total number of ACR criteria and SDI score at enrollment were higher in the $\mathrm{LN}$ group than in the non-LN group $(5.8 \pm 1.4$ vs. $4.7 \pm 1.2, \mathrm{p}<0.001$ and $0.4 \pm 0.9$ vs. $0.3 \pm 0.6, p=0.001)$. However, non-renal SDI scores were comparable between the two groups. The renal biopsy results are also summarized in Table 1 . In patients with LN, the most common histologic LN classification was class IV $(n=226,44.6 \%)$, and the number of patients grew to 256 (50.5\%) when classes IV and IV+V were combined. The mean activity index was $7.0 \pm 4.2$ (range, $0 \sim 18$ ) and the mean chronicity index was $1.7 \pm 1.6$ (range, $0 \sim 8$ ) in the first renal biopsy.

\section{Factors associated with the presence of $L N$}

Factors associated with the presence of $\mathrm{LN}$ are summarized in Table 2. Among the various clinical features and wGRS in the univariate analysis, younger age at diagnosis $(\mathrm{OR}=0.97, \mathrm{p}<0.001)$, male sex $(\mathrm{OR}=1.78, \mathrm{p}=0.013)$, longer duration between SLE diagnosis and enrollment $(\mathrm{OR}=1.06, \mathrm{p}<0.001)$, presence of a malar rash $(\mathrm{OR}=$ 1.51, $\mathrm{p}<0.001)$, pleuritis $(\mathrm{OR}=2.95, \mathrm{p}<0.001)$, pericarditis $(\mathrm{OR}=2.49, \mathrm{p}<0.001)$, hemolytic anemia $(\mathrm{OR}=1.43$, $\mathrm{p}=0.037)$, lymphopenia $(\mathrm{OR}=1.35, \mathrm{p}=0.020)$, thrombocytopenia $(\mathrm{OR}=1.42, \mathrm{p}=0.014)$, anti-dsDNA antibodies $(\mathrm{OR}=2.57, \mathrm{p}<0.001)$, anti-Sm antibodies $(\mathrm{OR}=1.90$, $\mathrm{p}<0.001$ ), low complement (OR=1.69, $\mathrm{p}<0.001)$, absence of aPL $(O R=1.39, p=0.015)$, and higher wGRS $(\mathrm{OR}=1.24, \mathrm{p}<0.001)$ were significantly associated with the presence of LN. Among aPL antibodies, negative anti-beta 2 glycoprotein I IgM antibodies (anti- $\beta 2$ GPI IgM, $\mathrm{OR}=2.98, \mathrm{p}<0.001$ ), negative anticardiolipin antibodies $(\mathrm{OR}=1.32, \mathrm{p}<0.001)$, and negative lupus anticoagulant $(\mathrm{OR}=1.61, \mathrm{p}=0.012)$ test results were associated with

Table 2. Associations between clinical features and the presence of lupus nephritis

\begin{tabular}{|c|c|c|c|c|c|c|}
\hline \multirow{2}{*}{ Variable } & \multicolumn{3}{|c|}{ Univariate analysis } & \multicolumn{3}{|c|}{ Multivariate analysis } \\
\hline & OR & $95 \% \mathrm{Cl}$ & p-value & OR* & $95 \% \mathrm{Cl}$ & p-value \\
\hline Age at diagnosis of SLE (yr) & 0.97 & $0.95 \sim 0.98$ & $<0.001$ & 0.97 & $0.96 \sim 0.99$ & $<0.001$ \\
\hline Male sex & 1.78 & $1.12 \sim 2.82$ & 0.013 & 1.51 & $0.91 \sim 2.50$ & 0.112 \\
\hline Duration between SLE diagnosis and enrollment (yr) & 1.06 & $1.03 \sim 1.10$ & $<0.001$ & 1.04 & $1.01 \sim 1.08$ & 0.024 \\
\hline wGRS & 1.24 & $1.12 \sim 1.38$ & $<0.001$ & 1.16 & $1.01 \sim 1.08$ & 0.012 \\
\hline Malar rash & 1.51 & $1.19 \sim 1.93$ & $<0.001$ & 1.08 & $0.81 \sim 1.43$ & 0.581 \\
\hline Pleuritis & 2.95 & $2.10 \sim 4.14$ & $<0.001$ & 2.44 & $1.65 \sim 3.60$ & $<0.001$ \\
\hline Pericarditis & 2.49 & $1.72 \sim 3.60$ & $<0.001$ & 1.62 & $1.05 \sim 2.50$ & 0.029 \\
\hline Hemolytic anemia & 1.43 & $1.02 \sim 2.00$ & 0.037 & 1.17 & $0.80 \sim 1.70$ & 0.425 \\
\hline Lymphopenia & 1.35 & $1.05 \sim 1.73$ & 0.020 & 1.23 & $0.94 \sim 1.62$ & 0.132 \\
\hline Thrombocytopenia & 1.42 & $1.07 \sim 1.88$ & 0.014 & 1.28 & $0.94 \sim 1.74$ & 0.125 \\
\hline Anti-dsDNA antibodies & 2.57 & $1.93 \sim 3.51$ & $<0.001$ & 2.22 & $1.59 \sim 3.10$ & $<0.001$ \\
\hline Anti-Sm antibodies & 1.90 & $1.40 \sim 2.59$ & $<0.001$ & 1.70 & $1.21 \sim 2.38$ & 0.002 \\
\hline Low complement & 1.69 & $1.28 \sim 2.22$ & $<0.001$ & 1.37 & $1.01 \sim 1.86$ & 0.043 \\
\hline Negative aPL antibodies & 1.39 & $1.07 \sim 1.81$ & 0.015 & 1.60 & $1.19 \sim 2.13$ & 0.002 \\
\hline Negative anti- $\beta 2$ GPI IgM antibodies & 2.98 & $1.58 \sim 5.21$ & $<0.001$ & & & \\
\hline Negative anti-cardiolipin antibodies & 1.47 & $1.08 \sim 2.00$ & 0.014 & & & \\
\hline Negative lupus anticoagulant & 1.61 & $1.11 \sim 2.34$ & 0.012 & & & \\
\hline
\end{tabular}

OR: odds ratio, OR*adjusted for age at diagnosis, sex, and the duration between SLE diagnosis and enrollment, $\mathrm{Cl}$ : confidence interval, SLE: systemic lupus erythematosus, wGRS: weighted genetic risk score, dsDNA: double-stranded deoxyribonucleic acid, Sm: Smith, aPL: antiphospholipid. p-values and OR are calculated by logistic regression analyses. p-values $<0.05$. 
LN. In the multivariate analysis model, younger age at SLE diagnosis $(\mathrm{OR}=0.97, \mathrm{p}<0.001)$, longer duration between SLE diagnosis and enrollment $(\mathrm{OR}=1.04$, $\mathrm{p}=0.024)$, higher wGRS $(\mathrm{OR}=1.16, \mathrm{p}=0.012)$, presence of pleuritis $(\mathrm{OR}=2.44, \mathrm{p}<0.001)$, pericarditis $(\mathrm{OR}=1.62$, $\mathrm{p}=0.029)$, anti-dsDNA antibodies $(\mathrm{OR}=2.22, \mathrm{p}<0.001)$, anti-Sm antibodies $(\mathrm{OR}=1.70, \mathrm{p}=0.002)$, low complement $(\mathrm{OR}=1.37, \mathrm{p}=0.043)$, and absence of aPL antibodies $(\mathrm{OR}=1.60, \mathrm{p}=0.002)$ were statistically significant associated factors.

\section{Relationships among the wGRS, serology, and the presence of $L N$}

Since serologic markers, such as anti-dsDNA antibodies, anti-Sm antibodies, low complement, absence of aPL antibodies, and the wGRS were independently associated with the presence of LN and since the wGRS is an inherited trait from birth, we assumed that the wGRS could be the exposure and that serologic markers could be mediators for the presence of $\mathrm{LN}$. All three of the relationships in Figure 1 were confirmed to be statistically significant (Table 3), and the statistical power of the p-values decreased when anti-Sm antibodies and low complement were added in step two (OR before adding anti-Sm antibodies in step two: 1.83 , OR after adding anti-Sm antibodies in step two: 1.16, OR before adding low complement in step two: 1.60, OR after adding low complement in step two: 1.17). Through Sobel's test, we found an estimate of 2.66 for anti-Sm antibodies and an estimate of 2.17 for low complement, which were statistically significant (Figure 2, standard error $[\mathrm{SE}]_{\mathrm{A}}$ for anti-Sm antibodies: $0.066, \mathrm{SE}_{\mathrm{A}}$ for low complement: $0.057, \mathrm{SE}_{\mathrm{B}}$ for anti-Sm antibodies: 0.157, $\mathrm{SE}_{\mathrm{B}}$ for low complement: 0.141). Based on these processes, anti-Sm antibodies and low complement appeared to have indirect effects on the relationship between the wGRS and LN.

\section{DISCUSSION}

In this study, we investigated factors associated with the presence of LN in patients with SLE. Younger age at diagnosis, longer duration between SLE diagnosis to enrollment, presence of pleuritis or pericarditis, presence or absence of certain serologic markers, and higher wGRS were independently associated with the presence of LN. In addition, anti-Sm antibodies and low complement mediated an indirect relationship between the wGRS and LN.

Several studies have reported associated clinical characteristics in patients with $\mathrm{LN}$, including younger age of SLE onset [3,28], presence of a malar rash [29], hemolytic anemia [28], and thrombocytopenia [29], and increased number of fulfilled ACR criteria [29], which are in line with the findings of our study. Previous studies have also reported an association between serologic markers including anti-Sm antibodies $[3,29]$ and anti-dsDNA antibodies [7], and the presence of LN. Our study revealed that anti-dsDNA antibodies, anti-Sm antibodies, low complement, and absent aPL antibodies were factors associated with the presence of $\mathrm{LN}$ after adjusting for age, sex, and duration from diagnosis of SLE to enrollment. Regarding the relationship between aPL antibodies and LN, inconsistent results have been reported [7,30-32]. One study reported that the anti- $\beta 2$ GPI IgM antibodies are protective against $\mathrm{LN}$ [32], which is in accordance with our results. The authors postulated that a lack of data on anti- $\beta 2$ GPI antibodies in past research may contribute the negative effect of aPL antibodies against $\mathrm{LN}$. This inconsistent association may be due to different study designs and definitions.

The genetic components of $\mathrm{LN}$ have been of recent interest in the field of rheumatology $[8,33,34]$. Previous research using transgenic mice produced to express HLA-DR3 and develop antinuclear antibodies, an-

Table 3. Relationships among serologic markers, wGRS, and the presence of lupus nephritis

\begin{tabular}{clccc}
\hline \hline & \multicolumn{1}{c}{ Variables } & OR & $\mathrm{Cl}$ & $\mathrm{p}$-value \\
\hline \multirow{2}{*}{ Step 1 } & wGRS and Anti-Sm antibodies & 1.27 & $1.11 \sim 1.46$ & $<0.001$ \\
& wGRS and low complement & 1.18 & $1.04 \sim 1.32$ & 0.008 \\
Step 2 & wGRS and lupus nephritis & 1.20 & $1.08 \sim 1.34$ & 0.001 \\
Step 3 & Anti-Sm antibodies and lupus nephritis & 1.18 & $1.06 \sim 1.31$ & 0.004 \\
& Low complement and lupus nephritis & 1.19 & $1.06 \sim 1.32$ & 0.002 \\
\hline
\end{tabular}

OR: odds ratio, Cl: confidence interval, wGRS: weighted genetic risk score, Sm: Smith, Step 1: association between the wGRS and serologic markers, Step 2: association between serologic markers and lupus nephritis, Step 3: association between the wGRS and lupus nephritis. p-values and OR are calculated by logistic regression analyses. p-values $<0.05$. 
ti-dsDNA antibodies, and glomerulonephritis revealed that HLA-DR3 plays a critical role in generating an autoimmune response to anti-Sm antibodies and progressing to LN [35]. In another study, complement component 4 genes, near the major histocompatibility complex locus, reportedly generated a seven-fold variation in SLE risk [36]. Furthermore, studies have reported that anti-Sm antibodies [5] and low complement [37] are not only risk factors for LN but might also help to predict treatment response $[38,39]$. Based on previous research, we could reasonably assume that there is an association between serologic markers, such as anti-Sm antibodies and low complement, and LN. In this study, we revealed that anti-Sm antibodies and low complement could be mediators in the relationship between the wGRS and LN through Sobel's test. The results of our study can be explained by the assumptions of a previous study on possibility of binding of anti-Sm antibodies and complement to the kidney structure in LN [40]. It is important to determine the genetic effect of organ involvement to comprehend the pathogenesis of SLE [8]. We expect that the results of our study on the wGRS can be useful for predicting the disease course in patients with SLE in the future.

This study has some crucial limitations including insufficient data regarding the treatment regimen and treatment adherence in patients with SLE. We were unable to analyze the information on treatment because of a lack of data before enrollment and complexities of the regimen during the long follow-up period. Therefore, we focused on data that were not easily altered by treatment or disease course.

Our study also had some major strengths. First, we included a large number of patients with SLE with a relatively long follow-up period because of good patient compliance. Second, we analyzed high-validity data including clinical, serologic, histologic, and wGRS that were collected by well-trained medical staff in our center. Finally, this is the first study to determine the interactions between the wGRS and LN through the mediation of serologic markers.

As not all of the pathogenic mechanisms of $\mathrm{LN}$ have been elucidated, it is impossible to clearly determine the genetic background and risk factors for the presence of LN in patients with SLE. Further studies are needed to provide precision medicine for patients with $\mathrm{LN}$.

\section{CONCLUSION}

Younger age at diagnosis, presence of pleuritis, pericarditis, anti-dsDNA antibodies, anti-Sm antibodies, low complement, absence of aPL antibodies, and higher wGRS were independently associated with the presence of LN in patients with SLE. Anti-Sm antibodies and low complement seemed to mediate the association between wGRS and the presence of LN.

\section{ACKNOWLEDGMENTS}

This work was supported in part by the Bio \& Medical Technology Development Program of the National Research Foundation (NRF), funded by the Ministry of Science \& ICT (NRF-2017M3A9B4050335) and by Hanyang University Institute for Rheumatology Research, Republic of Korea.

\section{CONFLIC OF INTEREST}

No potential conflict of interest relevant to this article was reported.

\section{AUTHOR CONTRIBUTIONS}

J.M.S. and D.K. designed this study and drafted the manuscript. G.Y.A. assessed the clinical data and analysis. Y.C.K. and J.L. collected clinical and genetic data, and performed the statistical analysis. Y.P. advised on appropriate statistical techniques, including mediation analysis. Y.K.L., T.H.L., D.J.P., and Y.J.S. collected clinical data and interviewed patients. E.H. and K.K. analyzed the genetic data. G.Y.A., S.Y.B., C.B.C., and H.S.L. assessed the study design validity. S.C.B. designed this study and critically reviewed the manuscript.

\section{REFERENCES}

1. Tsokos GC. Systemic lupus erythematosus. N Engl J Med 2011;365:2110-21.

2. Formiga F, Moga I, Pac M, Mitjavila F, Rivera A, Pujol R. Mild presentation of systemic lupus erythematosus in elderly patients assessed by SLEDAI. SLE Disease Activity Index. Lupus 1999;8:462-5.

3. Hanly JG, O'Keeffe AG, Su L, Urowitz MB, Romero-Diaz J, Gordon C, et al. The frequency and outcome of lupus nephritis: results from an international inception cohort study. Rheumatology (Oxford) 2016;55:252-62. 
4. Fiehn C, Hajjar Y, Mueller K, Waldherr R, Ho AD, Andrassy $\mathrm{K}$. Improved clinical outcome of lupus nephritis during the past decade: importance of early diagnosis and treatment. Ann Rheum Dis 2003;62:435-9.

5. Ntatsaki E, Isenberg D. Risk factors for renal disease in systemic lupus erythematosus and their clinical implications. Expert Rev Clin Immunol 2015;11:837-48.

6. Mavragani CP, Fragoulis GE, Somarakis G, Drosos A, Tzioufas AG, Moutsopoulos HM. Clinical and laboratory predictors of distinct histopathogical features of lupus nephritis. Medicine (Baltimore) 2015;94:e829.

7. Alba P, Bento L, Cuadrado MJ, Karim Y, Tungekar MF, Abbs I, et al. Anti-dsDNA, anti-Sm antibodies, and the lupus anticoagulant: significant factors associated with lupus nephritis. Ann Rheum Dis 2003;62:556-60.

8. Reid S, Alexsson A, Frodlund M, Morris D, Sandling JK, Bolin K, et al. High genetic risk score is associated with early disease onset, damage accrual and decreased survival in systemic lupus erythematosus. Ann Rheum Dis 2020;79: 363-9.

9. Chen LY, Shi ZR, Tan GZ, Han YF, Tang ZQ, Wang L. Systemic lupus erythematosus with and without a family history: a meta-analysis. Lupus 2018;27:716-21.

10. Kwon YC, Chun S, Kim K, Mak A. Update on the genetics of systemic lupus erythematosus: genome-wide association studies and beyond. Cells 2019;8:1180.

11. Yin X, Kim K, Suetsugu H, Bang SY, Wen L, Koido M, et al. Meta-analysis of 208370 East Asians identifies 113 susceptibility loci for systemic lupus erythematosus. Ann Rheum Dis 2020;80:632-40.

12. Igo RP Jr, Kinzy TG, Cooke Bailey JN. Genetic risk scores. Curr Protoc Hum Genet 2019;104:e95.

13. Dudbridge F. Power and predictive accuracy of polygenic risk scores. PLoS Genet 2013;9:e1003348.

14. Tan EM, Cohen AS, Fries JF, Masi AT, McShane DJ, Rothfield NF, et al. The 1982 revised criteria for the classification of systemic lupus erythematosus. Arthritis Rheum 1982;25:1271-7.

15. Joo YB, Park SY, Won S, Bae SC. Differences in clinical features and mortality between childhood-onset and adult-onset systemic lupus erythematosus: a prospective single-center study. J Rheumatol 2016;43:1490-7.

16. Hochberg MC. Updating the American College of Rheumatology revised criteria for the classification of systemic lupus erythematosus. Arthritis Rheum 1997;40: 1725.

17. Gladman DD, Ibañez D, Urowitz MB. Systemic lupus erythematosus disease activity index 2000. J Rheumatol 2002;29:288-91.

18. Gladman D, Ginzler E, Goldsmith C, Fortin P, Liang M, Urowitz $\mathrm{M}$, et al. The development and initial validation of the Systemic Lupus International Collaborating Clinics/ American College of Rheumatology damage index for systemic lupus erythematosus. Arthritis Rheum 1996;39:363-9.

19. Hill GS, Delahousse M, Nochy D, Rémy P, Mignon F, Méry $\mathrm{JP}$, et al. Predictive power of the second renal biopsy in lupus nephritis: significance of macrophages. Kidney Int 2001; 59:304-16.

20. Weening JJ, D'Agati VD, Schwartz MM, Seshan SV, Alpers CE, Appel GB, et al. The classification of glomerulonephritis in systemic lupus erythematosus revisited. Kidney Int
2004;65:521-30.

21. Austin HA 3rd, Muenz LR, Joyce KM, Antonovych TA, Kullick ME, Klippel JH, et al. Prognostic factors in lupus nephritis. Contribution of renal histologic data. Am J Med 1983;75:382-91.

22. Hill GS, Delahousse M, Nochy D, Tomkiewicz E, Rémy P, Mignon F, et al. A new morphologic index for the evaluation of renal biopsies in lupus nephritis. Kidney Int 2000;58: 1160-73.

23. Kim K, Bang SY, Lee HS, Okada Y, Han B, Saw WY, et al. The HLA-DR $\beta 1$ amino acid positions 11-13-26 explain the majority of SLE-MHC associations. Nat Commun 2014;5:5902.

24. MacKinnon DP, Fairchild AJ, Fritz MS. Mediation analysis. Annu Rev Psychol 2007;58:593-614.

25. Kroon FPB, Veenbrink AI, de Mutsert R, Visser AW, van Dijk KW, le Cessie S, et al. The role of leptin and adiponectin as mediators in the relationship between adiposity and hand and knee osteoarthritis. Osteoarthritis Cartilage 2019;27: 1761-7.

26. He P, Fan SY, Guan JQ, Song WJ, Obore N, Chen WQ, et al. Mediation analysis for the relationship between dyslipidemia and coronary artery disease via hypersensitive C-reactive protein in a case-control study. Coron Artery Dis 2020;31:613-9.

27. Sobel ME. Asymptotic confidence intervals for indirect effects in structural equation models. Sociol Methodol 1982; 13:290-312.

28. Mok CC, Kwok RC, Yip PS. Effect of renal disease on the standardized mortality ratio and life expectancy of patients with systemic lupus erythematosus. Arthritis Rheum 2013; 65:2154-60.

29. Reppe Moe SE, Molberg Ø, Strøm EH, Lerang K. Assessing the relative impact of lupus nephritis on mortality in a population-based systemic lupus erythematosus cohort. Lupus 2019;28:818-25.

30. Parodis I, Arnaud L, Gerhardsson J, Zickert A, Sundelin B, Malmström V, et al. Antiphospholipid antibodies in lupus nephritis. PLoS One 2016;11:e0158076.

31. Varela DC, Quintana G, Somers EC, Rojas-Villarraga A, Espinosa G, Hincapie ME, et al. Delayed lupus nephritis. Ann Rheum Dis 2008;67:1044-6.

32. Mehrani T, Petri M. IgM anti- $\beta 2$ glycoprotein I is protective against lupus nephritis and renal damage in systemic lupus erythematosus. J Rheumatol 2011;38:450-3.

33. Iwamoto T, Niewold TB. Genetics of human lupus nephritis. Clin Immunol 2017;185:32-9.

34. Chen L, Wang YF, Liu L, Bielowka A, Ahmed R, Zhang H, et al. Genome-wide assessment of genetic risk for systemic lupus erythematosus and disease severity. Hum Mol Genet 2020;29:1745-56.

35. Chowdhary VR, Dai C, Tilahun AY, Hanson JA, Smart MK, Grande JP, et al. A central role for HLA-DR3 in anti-Smith antibody responses and glomerulonephritis in a transgenic mouse model of spontaneous lupus. J Immunol 2015;195: 4660-7.

36. Kamitaki N, Sekar A, Handsaker RE, de Rivera H, Tooley K, Morris DL, et al. Complement genes contribute sex-biased vulnerability in diverse disorders. Nature 2020;582:577-81.

37. Reátegui-Sokolova C, Ugarte-Gil MF, Harvey GB, Wojdyla D, Pons-Estel GJ, Quintana R, et al. Predictors of renal damage in systemic lupus erythematous patients: data from a 
multiethnic, multinational Latin American lupus cohort (GLADEL). RMD Open 2020;6:e001299.

38. Ahn SS, Yoo BW, Song JJ, Park YB, Lee SK, Lee SW. Anti-Sm is associated with the early poor outcome of lupus nephritis. Int J Rheum Dis 2016;19:897-902.

39. Mok CC, Ying KY, Tang S, Leung CY, Lee KW, Ng WL, et al.
Predictors and outcome of renal flares after successful cyclophosphamide treatment for diffuse proliferative lupus glomerulonephritis. Arthritis Rheum 2004;50:2559-68.

40. Yung S, Chan TM. Autoantibodies and resident renal cells in the pathogenesis of lupus nephritis: getting to know the unknown. Clin Dev Immunol 2012;2012:139365. 